\title{
A Case of Staphylococcal Scalded Skin Syndrome in a 1-Year Old Boy with Otitis Media
}

\author{
Selami Aykut Temiz' (iD, illkay Özer ${ }^{1}$, Arzu Ataseven¹, Sıddıka Fındık² \\ 'Department of Dermatology, Necmettin Erbakan University Meram School of Medicine, Konya, Turkey \\ 2Department of Patholgoy, Necmettin Erbakan University Meram School of Medicine, Konya, Turkey
}

Cite this article as: Temiz SA, Özer I, Ataseven A, Fındık S. A Case of Staphylococcal Scalded Skin Syndrome in a 1-Year Old Boy with Otitis Media. J Emerg Med Case Rep 2018; 9(4): 71-4.

\begin{abstract}
Introduction: Staphylococcal scalded skin syndrome (SSSS), also known as Ritter's Disease, is a severe disease seen mostly in newborns and children aged $<5$ years, and the mortality rate might reach to $4 \%$ despite an appropriate treatment. Exfoliative toxins cause intraepidermal separation in the stratum granulosum and lead to a condition that is characterized with widespread epidermolysis all over the body and bullae with positive Nikolsky's sign. SSSS might be fatal due to loss of skin barrier. Immediate initiation of an anti-staphylococcal drug therapy is required for the treatment of this toxin-dependent disease, which is one of the emergencies of dermatology practices.
\end{abstract}

Case Report: A 1-year-old boy was referred to a dermatology clinic from pediatric emergency service with widespread epidermolysis all over the body, including erosion and bullous formation that had been appeared in the inguinal region. SSSS was suspected in this case, and skin biopsy was performed to confirm the diagnosis. He was hospitalized in the Pediatric Infectious Diseases Service, and treatment comprising intravenous administration of (i.v.) vancomycin (40 mg/kg/day) and 3 gIVIG ( intravenous immunoglobulin) was initiated.

Conclusion: Staphylococcus aureus, a rather rare agent of otitis media, is remarkable in terms of causing the complication of SSSS, which is very rare. In our case report, we aimed to remark to this rare condition (association of otitis media and SSSS).

Keywords: Staphylococcal scalded skin syndrome, Staphylococcus aureus, otitis media

Received: 22.12.2017 Accepted: 22.02.2018

\section{Introduction}

Staphylococcal scalded skin syndrome (SSSS), also known as Ritter's Disease, is a staphylococcal exfoliative toxin-mediated disease initiated by fever, irritability, and sensitivity in the skin after a local infection, such as upper respiratory tract infection, bullous impetigo, otitis media, and purulent conjunctivitis mostly in newborns and children aged $<5$ years, that is frequently caused by Staphylococci (1). The mortality rate might reach to $4 \%$ despite an appropriate treatment (1). This illness is rarely observed in adults with immune deficiency or chronic renal insufficiency.

This syndrome was first described by Ritter in 1878, and Lyell mentioned about SSSS in the case reports published in 1956 (2). Nowadays, SSSS is clearly distinguished from other common epidermal necrolysis syndromes and the term "Ritter" is used for SSSS in newborns.

Staphylococcus aureus is a common microbial species that is permanently colonized on the epidermis surrounding the nostrils, and it is generally associated with temporal flora and occasionally causes infections in $20 \%$ of the population. The nasal mucosa,

This study was presented at the $26^{\text {th }}$ Turkish National Congress of Dermatology, 2016

Address for Correspondence:

Selami Aykut Temiz, Department of Dermatology, Necmettin Erbakan University Meram School of Medicine, Konya, Turkey

E-mail: aykutmd42@gmail.com

oCopyright 2018 by Emergency Physicians Association of Turkey - Available online at www.jemcr.org 
conjunctival mucosa, or umbilical cord might be colonized with $S$. aureus without causing infection (3). SSSS has also been reported in a newborn with chorioamnionitis (4).

Staphylococcus aureus (S. aerius) phage group II (type 3A, 3B, 5S, 71) is usually responsible for this illness (3). Exfoliative toxins (exfoliative toxins $A$ and B) cause intraepidermal separation in the stratum granulosum and lead to a condition that is characterized by widespread epidermolysis all over the body and bullae with a positive Nikolsky's sign. SSSS might be fatal due to secondary infections, hypothermia, dehydration, and liquid-electrolyte imbalance associated with loss of skin barrier. It is recommended to immediately initiate anti-staphylococcal drug therapy for the treatment of toxin-dependent disease, which is one of the emergencies of dermatology practices (1).

We report the case of a 1-year-old boy who developed SSSS after acute otitis media infection.

\section{Case Report}

A 1-year-old boy was referred to the dermatology clinic from pediatric emergency service with widespread epidermolysis all over the body, including erosion and bullous formation that had appeared in the inguinal region 4 days previously. In his medical history, he was diagnosed with otitis media a week ago and received amoxicillin-

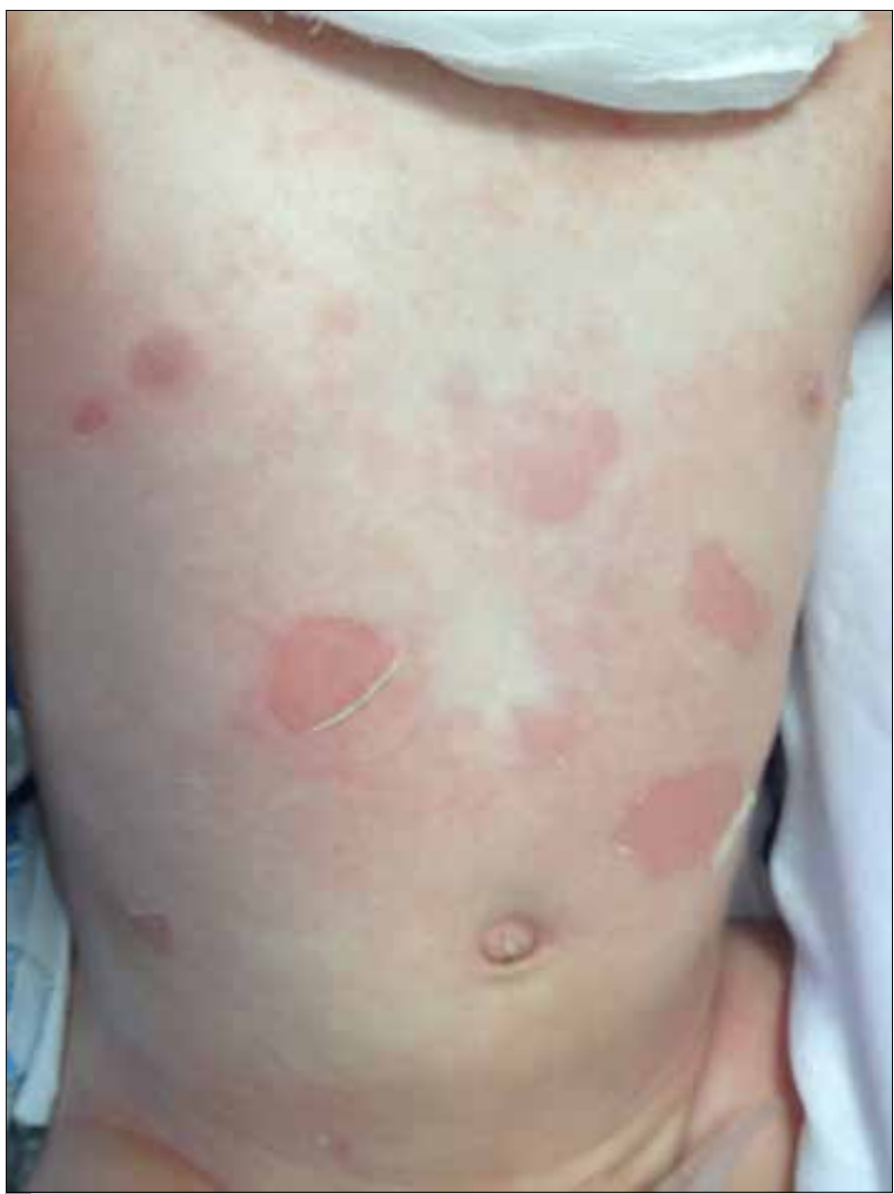

FIGURE 1. Extensive exfoliation was present in his abdomen, dorsum, inguinal region, lateral body sides, and extremities. clavulanate therapy. There was no special characteristic in his medical and family background. His physical examination revealed clear consciousness, moderate health condition, anxiousness, and irritableness. His had fever (temperature $39.1^{\circ} \mathrm{C}$ ). Otoscopic examination performed by the Ear Nose Throat department suspected acute otitis media. In his dermatological examination, extensive exfoliation was present in his abdomen, dorsum, inguinal region, lateral body sides, and extremities (Figure 1), and eroded and exfoliative lesions were present in his face, lips, and eyelids (Figure 2). He was hospitalized in Pediatric Infectious Diseases Service, and intravenous (i.v.) vancomycin (40 mg/kg/day) and $3 \mathrm{gr}$ IVIG (single dose) were initiated.

His laboratory findings were as follows: urea level: $38 \mathrm{mg} / \mathrm{mL}$, creatinine level: $0.72 \mathrm{mg} / \mathrm{dL}$, CRP level: $2 \mathrm{mg} / \mathrm{L}$, TSH level: $7.44 \mathrm{mIU} /$ mL, WBC: 10700/uL, neu: 8550/uL, and Hgb: 14.4 g/dL.SSSS was suspected in this case, and skin biopsy was performed to confirm the diagnosis. Histopathological examination showed separation in the subcorneal multilayer squamous epithelium, mild acanthosis, and inflammatory mononuclear cell infiltration containing isolated eosinophils in the perivascular region of papillary dermis (Figure 3; H\&E40X). The direct immunofluorescence examination did not show any findings. The subcorneal separation and absence of fullthickness necrosis were diagnosed as histopathological SSSS. Informed consent form was obtained from the relatives of patients.

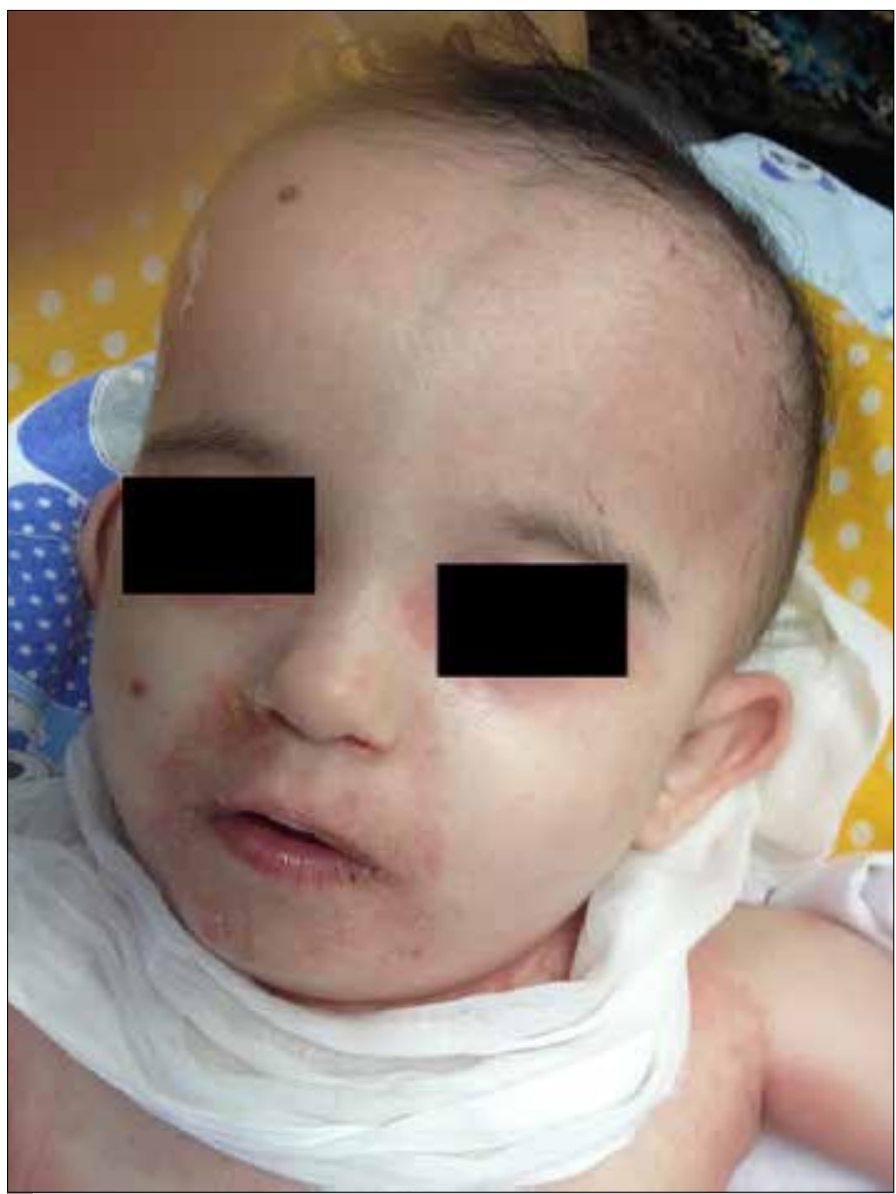

FIGURE 2. Eroded and exfoliative lesions were present in his face, lips, and eyelids. 


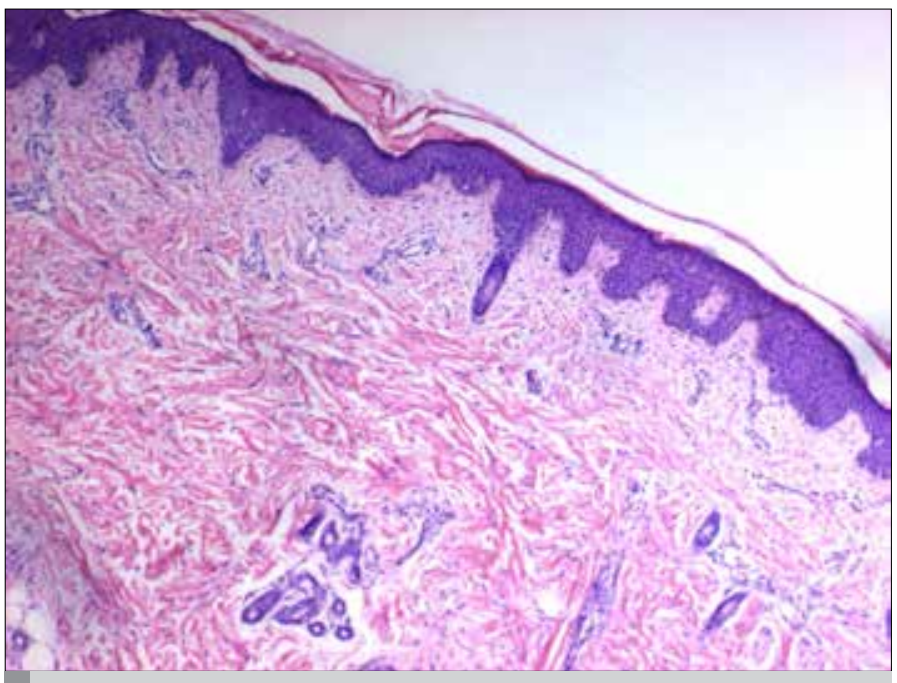

FIGURE 3. The histopathological examination showed separation in the subcorneal multilayer squamous epithelium, mild acanthosis and inflammatory mononuclear cell infiltration containing isolated eosinophils in the perivascular region of papillary dermis.

His erythema and erosion regressed during the first week, and he was discharged from the hospital with complete recovery after 20 days.

\section{Discussion}

Acute otitis media (AOM) is the inflammation of the middle ear that is progressed as a simple upper respiratory tract infection (UPTI) extending from the nasal cavity with the sudden development of local and systemic symptoms (5). It is the second most common infection in children after UPTI, and $90 \%$ of the children aged $<2$ years have AOM at least once (5). Streptococcus pneumonia is the most frequent cause of AOM, and it is followed by Haemophilus influenzae and Moraxella catarrhalis. The distribution of these agents are as follows: $S$. pneumonia (30\%-50\%), H. influenzae (20\%-30\%), and M. catarrhalis (10\%-20\%). They are followed by Group A Streptococcus (approximately up to 5\%), S. aureus( $<\% 1)$, and other agents (5). In our patient, the infectious agent is $S$. aureusthatis rarely seen in AOM.

Mortality in SSSS is generally associated with delayed diagnosis, use of inappropriate antibiotics, secondary infections, pneumonia, water-electrolyte imbalance, and sepsis (1). Toxic epidermal necrosis (TEN), Stevens-Johnson syndrome, toxic shock syndrome, Kawasaki disease, epidermolysisbullosa, and bullosa impetigo should be considered for the differential diagnosis of SSSS-suspicious patients (1).

While systemic glucocorticosteroids are generally preferred in drug eruptions involving TEN, they are contraindicative in SSSS (1). Therefore, a differential diagnosis is highly important. Bullae with a positive Nikolsky's sign is present in TEN likewise, mucosal lesions accompany in all conscience. The absence of mucosal lesions in SSSS might be helpful in the diagnosis (1). Moreover, histopathological examination revealed dermoepidermal separation in TEN and necrosis is present throughout the epidermis (1).
Although SSSS and pemphigus foliaceus are irrelevant diseases, the target antigen has been detected as desmoglein-1 for both in 2000s (6). The detection of target antigen was clarified the absence of mucosal involvement in SSSS.

Clinical diagnosis is generally preferred; however, a skin biopsy is the method for final diagnosis. Lesions are clinically initiated from skinfold areas, such as the axillary and groin (1). SSSS is caused by Group 2 staphylococcus epidermolytic (exfoliative) exotoxins A and B. Exfoliative toxins $A$ and $B$ are responsible for acantholytic infection and serine proteases is resulted in epidermolysis (7). These toxins target desmoglein-1 that provides intercellular interactions of keratocytes, and thus cause the loss of intracellular connection, epidermal separation (stratum granulosum), and extension of the inflammation (7).

Patients with SSSS usually treated by hospitalization. Tissue samples for culture must be obtained from skin and nasopharyngeal mucosa. As bullous toxins are produced, it is not meaningful to obtain samples from bullous lesions. In addition to systemic antibiotic therapy, it is important to provide protection against secondary infections. Moreover, the regulation of fluid-electrolyte balance is required for the treatment, as well. Since penicillinase is produced by the ratios of 95\% in S. aureus, I.V. resistant anti-staphylococcal antibiotics must be administered to the patients. The combination therapies of methicillin (resistant to penicillinase) or beta-lactamase inhibitors (clavulanic acid, sulbactam, and tazobactam) with penicillin are the first preference (1). Nowadays, methicillin-resistant staphylococcus aureus (MRSA) is detected at increasing rates (8). In a study performed in the USA, the rate of MRSA was detected over $60 \%$ in the intensive care units (8). In recent epidemiological studies, it has been shown that MRSA is not only limited to the intensive care units, but also it has been gradually increased in the community $(9,10)$. In our case study, vancomycin was added to our patient with unremitting acute otitis despite of amoxicillin/clavulanic acid treatment. In the literature, the administration of intravenous immunoglobulin ( $0.4 \mathrm{~g} / \mathrm{kg} /$ day, for 5 days) was found to be effective in certain cases (1). We administered i.v.vancomycin, local wound therapy, and single-dose IV immunoglobulin (3 g) to our patient, and all lesions were regressed in a week.

\section{Conclusion}

We want to draw attention to the fact that common infectious diseases can lead to dermatological emergencies. In our case report, we aimed to remark that $\mathrm{S}$. aureus, a rare microbial agent causing AOM, might cause SSSS in children.

Informed Consent: Written informed consent was obtained from patient's parent who participated in this study.

Peer-review: Externally peer-reviewed.

Author Contributions: Concept- I.Ö., S.A.T.; Design - A.A.,I.Ö.,S.A.T.; Supervision- I.Ö.,A.A.S.F.; Resources - I.Ö.,S.A.T.; Materials - I.Ö., S.A.T.; Data Collection and/orProcessing-S.A.T.; Analysis and/orInterpretation- I.Ö.; LiteratureSearchA.A.,S.A.T.; WritingManuscript- S.A.T.; Critical Review- I.Ö.; Other- S.F.

Conflict of Interest: The authors have no conflict of interest to declare. 
Financial Disclosure: The authors declared that this study has received no financial support.

\section{References}

1. Handler MZ, Schwartz RA. Staphylococcalscalded skin syndrome: diagnosisandmanagement in childrenandadults. J Eur Acad Dermatol Venereol 2014; 28: 1418-23. [CrossRef]

2. Lyell A. Toxicepidermalnecrolysis: An eruptionresemblingscalding of the skin. Br J Dermatol 1956; 68: 355-61. [CrossRef]

3. Jenkins A, Diep BA, Mai TT, Vo NH, Warrener P, Suzich J, et al. Differential expression and roles of Staphylococcus aureus virulence determinants during colonization and disease. MBio 2015; 6: e02272-14. [CrossRef]

4. Loughead JL. Congenitalstaphylococcalscalded skin syndrome: Report of a case. Pediatr Infect Dis J 1992; 11: 413-4. [CrossRef]
5. Neff MJ; American Academy of Pediatrics; American Academy of FamilyPhysicians. AAP, AAFP releaseguideline on diagnosisandmanagement of acuteotitismedia. Am Fam Physician 2004; 69: 2713-5. [CrossRef]

6. Amagai M. Desmoglein as a target in autoimmunity and infection. J Am Acad Dermatol 2003; 48: 244-52.

7. Blyth M, Estela C, Young AE. Severe staphylococcalscalded skin syndrome in children. Burns 2008; 34: 98-103. [CrossRef]

8. Hawkey PM. Thegrowing burden of antimicrobial resistance. J Antimicrob Chemother 2008; 62(Suppl 1): i1-9. [CrossRef]

9. Stryjewski ME, Corey GR. New treatments for methicillin-resistant Staphylococcus aureus. Curr Opin Crit Care 2009; 15: 403-12. [CrossRef]

10. Soriano A, Marco F, Martínez JA, Pisos E, Almela M, Dimova VP, et al Influence of vancomycin minimum inhibitoryconcentration on thetreatment of methicillin-resistantStaphylococcusaureusbacteremia. Clin Infect Dis 2008; 46: 193-200. 